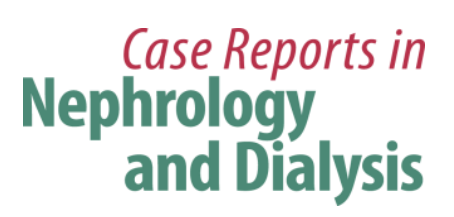

Case Rep Nephrol Dial 2017;7:1-5

\title{
Unsuccessful Treatment with Abatacept in Recurrent Focal Segmental Glomerulosclerosis after Kidney Transplantation
}

\author{
Tilde Kristensen Per Ivarsen Johan Vestergaard Povlsen \\ Department of Renal Medicine, Aarhus University Hospital, Aarhus, Denmark
}

\section{Keywords}

Focal segmental glomerulosclerosis - Abatacept - Recurrent focal segmental glomerulosclerosis · B7-1 inhibitor

\begin{abstract}
Recurrence of focal segmental glomerulosclerosis (FSGS) after renal transplantation occurs in up to $20-50 \%$ of FSGS patients and is associated with inferior allograft survival. Treatment of both primary FSGS as well as recurrent FSGS after transplantation with plasma exchange and immunosuppression is often unsuccessful and remains a major challenge as the disease still leads to end-stage renal disease and decreased graft survival. Previous case reports have described patients with recurrent FSGS who were successfully treated with a B7-1 inhibitor (abatacept) inducing partial or complete remission. The rational basis for believing in abatacept as a new therapeutic drug for the treatment of FSGS is the study by Yu et al. [N Engl J Med 2013;369:2416-2423] showing B7-1 in immunostainings of the podocytes. The authors speculated that B7-1 immunostaining of renal biopsies might identify a subgroup of patients who would benefit from abatacept treatment. We present a case with recurrent FSGS after renal transplantation. The patient was unsuccessfully treated with B7-1 inhibitors. Although the patient was treated with abatacept $10 \mathrm{mg} / \mathrm{kg}$ body weight twice, the proteinuria and decreased graft function remained unchanged, and he never reached remission.
\end{abstract}




\section{Case Reports in Nephrology and Dialysis}

\section{Introduction}

The pathogenesis of focal segmental glomerulosclerosis (FSGS) is not completely understood, but both abnormalities in T and B cells leading to podocyte injury [1] and the existence of a permeability factor which might be able to damage the podocytes [2] have been suggested. Recurrence of FSGS after renal transplantation occurs in up to 20-50\% [3] of FSGS patients and is associated with inferior allograft survival. Treatment of both primary FSGS as well as recurrent FSGS after transplantation with plasma exchange (PE) and immunosuppression is often unsuccessful [4]. In a recent study, 5 patients with recurrent FSGS were successfully treated with a B7-1 inhibitor (abatacept), inducing partial or complete remission [5]. In immunostainings, the authors found B7-1 in the renal biopsies and suggested that it might be a useful biomarker for the treatment of FSGS and a new therapeutic target. B7-1 is believed to be implicated in the development of proteinuria in general [6].

Here, we present a case with recurrent FSGS after renal transplantation. The patient was unsuccessfully treated with B7-1 inhibitors.

\section{Case Presentation}

A 48-year-old Caucasian man was hospitalized with nephrotic syndrome in March 2014. Serum creatinine was $457 \mu \mathrm{mol} / \mathrm{L}$, plasma albumin $9 \mathrm{~g} / \mathrm{L}$, and 24 -h proteinuria $13.3 \mathrm{~g} / \mathrm{day}$. A kidney biopsy was consistent with minimal-change nephropathy, but high-dose prednisolone and rituximab twice did not induce remission. In April 2014, he became anuric and started hemodialysis. In May 2015, he received a kidney transplant (living related donor, mismatch 1:1). Before transplant and on day 1 he received Thymoglobulin as induction therapy. Afterwards, he was immunosuppressed with tacrolimus and mycophenolate mofetil. He had immediate onset of graft function, but on day 1 after transplantation the albuminuria-tocreatinine ratio was $2,907 \mathrm{mg} / \mathrm{g}$. On day 4 , he had a 24 -h albuminuria of $2.7 \mathrm{~g} /$ day, and the creatinine was rising. A graft biopsy showed no signs of rejection but was suspected of FSGS with moderate to severe podocyte fusion seen on electron microscopy. Treatment with PE at $4 \mathrm{~L} /$ session daily for 3 days and every second day for the next 14 days was started. Immunoglobulin (150 mg/kg body weight) was given after each PE. The degree of albuminuria remained stable despite $\mathrm{PE}$, and estimated glomerular filtration rate was stable around 50 $\mathrm{mL} / \mathrm{min}$. PE was continued 3 times per week. Forty days after transplantation, he was started on an angiotensin-receptor blocker and continued PE once weekly, and a second graft biopsy showed moderate to severe podocyte fusion. After 60 days, the albuminuria increased, and PE was intensified to twice weekly. On day 105 and 140 after the transplantation, the patient was treated with abatacept $10 \mathrm{mg} / \mathrm{kg}$ body weight, but proteinuria and graft function remained unchanged. As the patient experienced severe side effects from the abatacept treatment (leukopenia and dyspnea), no further doses were planned. Due to persisting albuminuria in the nephrotic range and slowly declining graft function, a third graft biopsy was performed on day 450 showing classical signs of FSGS (Fig. 1, Fig. 2). Genetic testing for FSGS showed no known mutations. 


\section{Case Reports in Nephrology and Dialysis}

\section{Discussion}

Although the present patient initially was diagnosed with minimal-change nephropathy, the course and the latest biopsy clearly showed that he was suffering from recurrent FSGS shortly after kidney transplantation. Initially, the patient was steroid resistant. After transplantation, the patient was enrolled in a protocol and randomized to steroid-free immunosuppression. Due to a lack of evidence of steroids inducing remission in recurrent FSGS, no steroid treatment was started.

Treatment with a B7-1 inhibitor was ineffective and did not induce remission. Therapeutic regiments with $\mathrm{PE}$, angiotensin-receptor blocker, and immunosuppression were ineffective as well but have been described to induce remission in up to $60 \%$ of patients with FSGS [3]. Failure of treatment with a B7-1 inhibitor in adults and children has been described previously [7-11]. The rational basis for believing in abatacept as a new therapeutic drug for the treatment of FSGS is the study by Yu et al. [5] showing B7-1 in immunostainings of the podocytes. The authors speculated that B7-1 immunostaining of renal biopsies might identify a subgroup of patients who would benefit from abatacept treatment and presented 5 cases in which the patients gained partial or complete remission. Later, a prospective study of 9 patients with FSGS described no remission after abatacept [8]. All patients were B7-1 immunostaining negative, and the authors concluded that B7-1 was not expressed in podocytes. This was confirmed both in an independent validation cohort of 22 patients with recurrent FSGS after transplantation and in a cohort of patients with other proteinuric diseases in native kidneys. Both abatacept and belatacept, which has a 10 -fold increased capacity of B7-1 inhibition compared to abatacept, were tested. Still, new case reports on successful treatment with abatacept in recurrent FSGS have been published [12]. Due to a lack of evidence, treatment with calcineurin inhibitors was not considered for the present patient. Treatment of FSGS and recurrent FSGS after transplantation remains a major challenge as the disease still leads to end-stage renal disease and decreased graft survival, and the role for abatacept remains unclear.

\section{Acknowledgement}

Dr. Søren Rasmus Palmelund Krag, Department of Pathology, Aarhus University Hospital, is acknowledged for the biopsy photo.

\section{Statement of Ethics}

The authors have no ethical conflicts to declare.

\section{Disclosure Statement}

The authors have no conflicts of interest to disclose. 


\section{Case Reports in Nephrology and Dialysis}

\section{References}

$\checkmark 1$ Cara-Fuentes G, Kairalla JA, Ishimoto T, Rivard C, Johnson RJ, Garin EH: Rituximab in idiopathic nephrotic syndrome: does it make sense? Pediatr Nephrol 2014;29:1313-1319.

2 Cravedi P, Kopp JB, Remuzzi G: Recent progress in the pathophysiology and treatment of FSGS recurrence. Am J Transplant 2013;13:266-274.

-3 Ponticelli C: Recurrence of focal segmental glomerular sclerosis (FSGS) after renal transplantation. Nephrol Dial Transplant 2010;25:25-31.

-4 Malaga-Dieguez L, Bouhassira D, Gipson D, Trachtman H: Novel therapies for FSGS: preclinical and clinical studies. Adv Chronic Kidney Dis 2015;22:e1-e6.

5 Yu CC, Fornoni A, Weins A, et al: Abatacept in B7-1-positive proteinuric kidney disease. N Engl J Med 2013;369:2416-2423.

-6 Kronbichler A, Leierer J, Oh J, Meijers B, Shin JI: Immunologic changes implicated in the pathogenesis of focal segmental glomerulosclerosis. Biomed Res Int 2016;2016:2150451.

-7 Grellier J, Del BA, Milongo D, Guilbeau-Frugier C, Rostaing L, Kamar N: Belatacept in recurrent focal segmental glomerulosclerosis after kidney transplantation. Transpl Int 2015;28:1109-1110.

-8 Delville M, Baye E, Durrbach A, et al: B7-1 blockade does not improve post-transplant nephrotic syndrome caused by recurrent FSGS. J Am Soc Nephrol 2016;27:2510-2527.

9 Jayaraman VK, Thomas M: Abatacept experience in steroid and rituximab-resistant focal segmental glomerulosclerosis. BMJ Case Rep 2016, DOI: 10.1136/bcr-2016-214396.

10 Alkandari O, Nampoory N, Nair P, et al: Recurrent focal segmental glomerulosclerosis and abatacept: case report. Exp Clin Transplant 2016;14:456-459.

11 Garin EH, Reiser J, Cara-Fuentes G, et al: Case series: CTLA4-IgG1 therapy in minimal change disease and focal segmental glomerulosclerosis. Pediatr Nephrol 2015;30:469-477.

-12 Sprenger-Mahr H, Zitt E, Soleiman A, Lhotta K: Successful treatment of focal segmental glomerulosclerosis after kidney transplantation with plasma exchange and abatacept in a patient with juvenile rheumatoid arthritis. Case Rep Transplant 2016;2016:7137584.

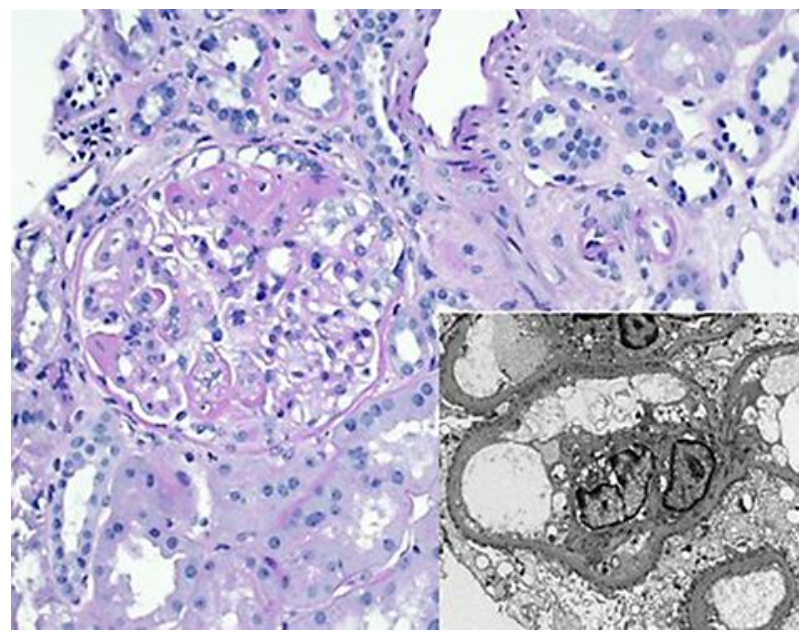

Fig. 1. Light microscopy of the renal biopsy showing focal segmental glomerulosclerosis. Inset Electron microscopy of the renal biopsy showing podocyte foot process fusion. 


\section{Case Reports in Nephrology and Dialysis}

(c) 2016 The Author(s). Published by S. Karger AG, Basel DOI: $10.1159 / 000454947$ www.karger.com/cnd

Kristensen et al.: Unsuccessful Treatment with Abatacept in Recurrent Focal Segmental Glomerulosclerosis after Kidney Transplantation

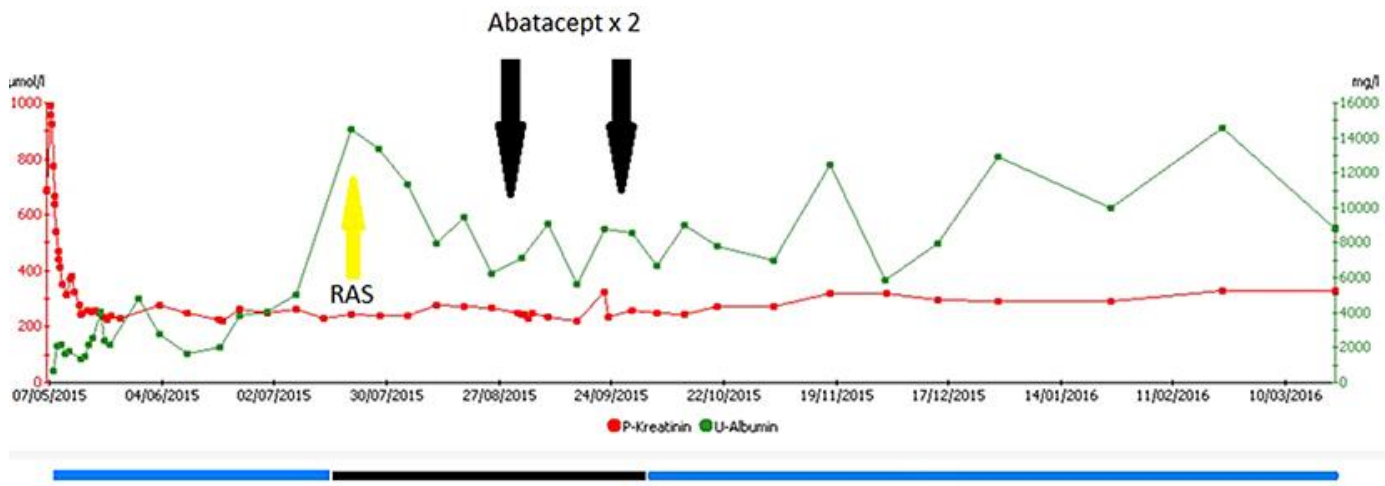

PE $\times 2-3 /$ week

PE $\times 1-2 /$ week

$\mathrm{PE} \times 1 /$ week

Fig. 2. Time line of albuminuria and plasma creatinine in relation to therapeutic interventions. RAS, reninangiotensin system inhibitor; PE, plasma exchange. 